\title{
Application of the incremental hole-drilling method for the determination of residual stresses in glass-fiber reinforced composites
}

\author{
Frank Schweizer ${ }^{1, a^{*}}$, Marius Dickele ${ }^{1, b}$ and Michael Luke ${ }^{1, c}$ \\ ${ }^{1}$ Fraunhofer IWM, Wöhlerstr. 11, 79108 Freiburg, Germany \\ afrank.schweizer@iwm.fraunhofer.de, ${ }^{b}$ marius.dickele@iwm.fraunhofer.de, \\ cmichael.luke@iwm.fraunhofer.de
}

Keywords: Hole drilling method, Reinforced composites, Residual stresses, Finite Element Analysis, Calibration coefficients

\begin{abstract}
The incremental hole-drilling method was adapted for the measurement of residual stresses in polymer based composite materials. The parameters of the hole drilling process and the strain measurement in non-reinforced and 70 weight-percent glass-fiber-reinforced polypropylene were investigated. Calibration coefficients for the calculation of the residual stresses in the orthotropic material systems were determined by Finite Element Analysis. By means of application of the method to unidirectional and cross-ply glass-fiber reinforced composites the residual stresses, maximum measurable depths and measurement uncertainties could be derived.
\end{abstract}

\section{Introduction}

In the manufacturing process of composite materials residual stresses may arise inside of the components. This happens particularly while cooling unidirectional and cross-ply reinforced tapes to room temperature, because of the differing thermal expansion of the fibers and the matrix. The evolution of deformations and residual stresses in the components reduce the performance of the material in the application.

For the residual stress estimation in the outer layer of engineering materials the hole-drilling method is a well-established technique [1] and also a promising technique for composite materials [2]. Applying the hole-drilling method a circular hole is drilled into the surface of a specimen. The relieved strains in the surrounding of the hole can be measured by strain gages or optical methods.

Using the measured strain data and experimentally or numerically determined coefficients the residual stresses in the material can be calculated. This can also be done incrementally. Drilling the hole in small steps into the material can be used to determine the change of the residual stresses as a function of the depth in the outer layer of the material.

The hole-drilling method is mostly used in metallic components. For new material systems the different influences on the hole-drilling process and the evaluation of the residual stresses have to be adapted. In this work the adaption of the incremental hole-drilling method for polypropylene based components and composites made of 70 weight-percent glass-fibers is presented. Because of the orthotropic material properties of the unidirectional and cross-ply reinforced composites, the procedures for the determination of calibration coefficients had to be adapted as well. 


\section{Experimental Procedure}

\section{Components used for the adaption of the hole-drilling procedure}

In the study the following structures shown in Figure 1 were used to evaluate the hole-drilling method:

(1) Pure Polypropylene (PP)

(2) Cross-ply laminate with the stacking sequence $[0,90,0,90] \mathrm{s}$ with a layer thickness of $0.25 \mathrm{~mm}$ made of PP 70 weight-percent reinforced composite (PP/GF70 cross-ply laminate)

(3) Reinforced composite made of $2.0 \mathrm{~mm}$ PP and $2.0 \mathrm{~mm}$ unidirectional GF70 with fiber direction parallel to the long axis of the component (PP/GF70-laminate)

(4) Reinforced composite made of $2.0 \mathrm{~mm}$ PP and $2.0 \mathrm{~mm}$ unidirectional GF70, with fiber direction perpendicular to the long axis of the component

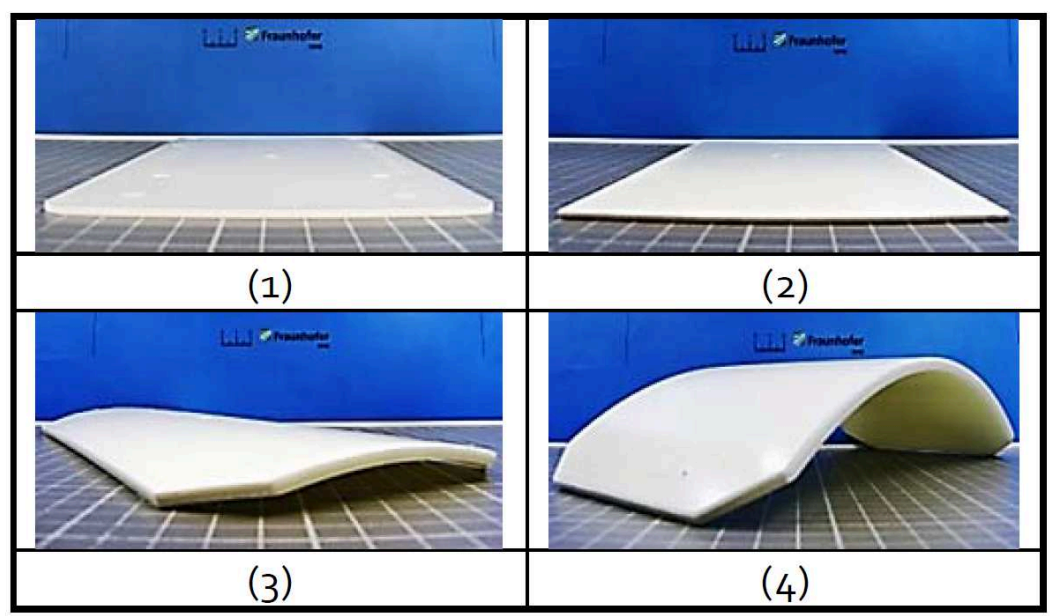

Fig.1 Evaluated components: (1) PP, (2) PP/GF70-Cross-ply laminate, (3) PP/GF70 Unidirectional laminate with fiber direction parallel to the long axis of the component, (4) PP/GF70

Unidirectional laminate with fiber direction perpendicular to the long axis of the component

The deformation of the unidirectional reinforced laminates which is due to the differing thermal expansion of the components and caused by the production process becomes already visible when the manufacturing tool is opened.

To measure the residual stress state three strain gage rosettes of type Vishay MicroMeasurement CEA-06-062UM-120 were glued to the surface of every component. As shown in Fig. 2a every strain gage rosette consists of three single strain gages surrounding the drilled hole in the axially aligned center. The rosettes have an offset of $45^{\circ}$ to each other. The orientation of one of the two outer strain gages was always aligned to the assumed fiber direction of the components. The diameter of the hole was $1.6 \mathrm{~mm}$ drilled by a milling cutter of type VHM-ZOX $1.5 \mathrm{~mm}$ GARANT. The holes in the unidirectional laminates were always drilled in the polypropylene part (PP-side).

\section{Measurement uncertainty and influence of the drilling speed}

A suitable drilling speed for the given material system was evaluated using a light-optical microscope to compare the geometry of the wall and the bottom of the drilled holes. At speeds of 500, 5000 and $6000 \mathrm{rpm}$, only small differences in the geometry and surface of the holes could be identified. At a low drilling speed of $20 \mathrm{rpm}$ misalignments of the hole geometry could be examined.

For the evaluation of the uncertainties of the drilling procedure three independent holes were drilled in every component at a speed of $5000 \mathrm{rpm}$. The maximum deviations from the mean values 
of the three measurements per component are shown in Fig. $2 b$ for all of the three strain gages of the rosettes at a drilling depth of $0.9 \mathrm{~mm}$. The measurement error of the released strains is quiet large, especially in the cross-ply laminate and in one direction of the pure polypropylene. This is because the error value of all material systems is of the same order, but as can be seen in Fig. 5 a-d the released strains in these two cases are much lower.

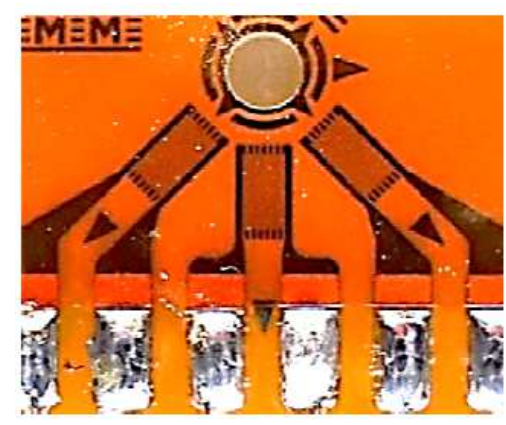

Fig. 2a Drilled Hole in Strain Gage Rosette (Vishay Micro Measurement CEA-06-062-UM)

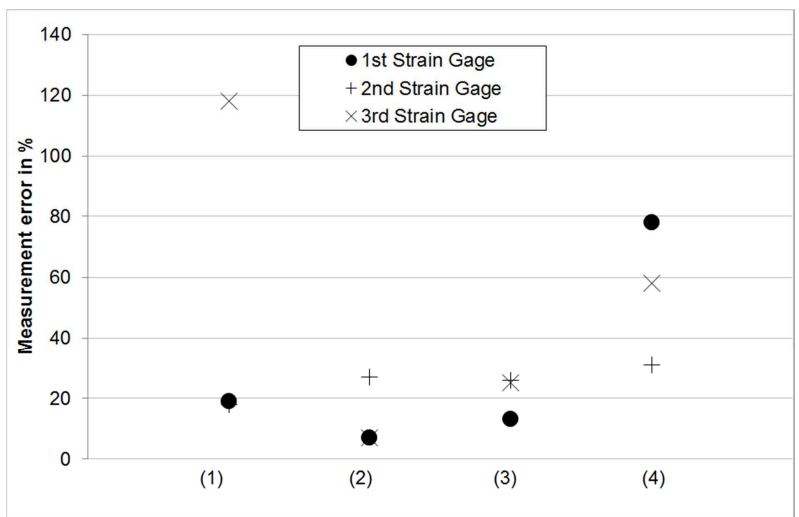

Fig $2 \mathrm{~b}$ Maximum deviations of three repeated measurements: (1) PP, (2) Cross-ply laminate PP/GF70, (3) Unidirectional laminate PP/GF70 with fiber direction parallel to the long axis of the component, (4) Unidirectional laminate PP/GF70 with fiber direction perpendicular to the long axis of the component

Influence of the excitation voltage Because polymer based composite materials act as thermal isolators the influence of the strain gage excitation voltage had to be investigated. Therefore thermocouples of type $\mathrm{K}$ were glued on different positions:

- Directly on the strain gage rosette which was mounted on polypropylene (Worst case)

- In the polypropylene material, directly under the gage position using a drilled hole from the opposite side.

- Directly on the strain gage rosette which was mounted on the PP/GF70 (Worst case)

- In the PP/GF70 material, directly under the gage position using a drilled hole from the opposite side.

In Figure 3 the temperature rise for different excitation voltage levels of the strain gages and the resulting temperatures are shown.

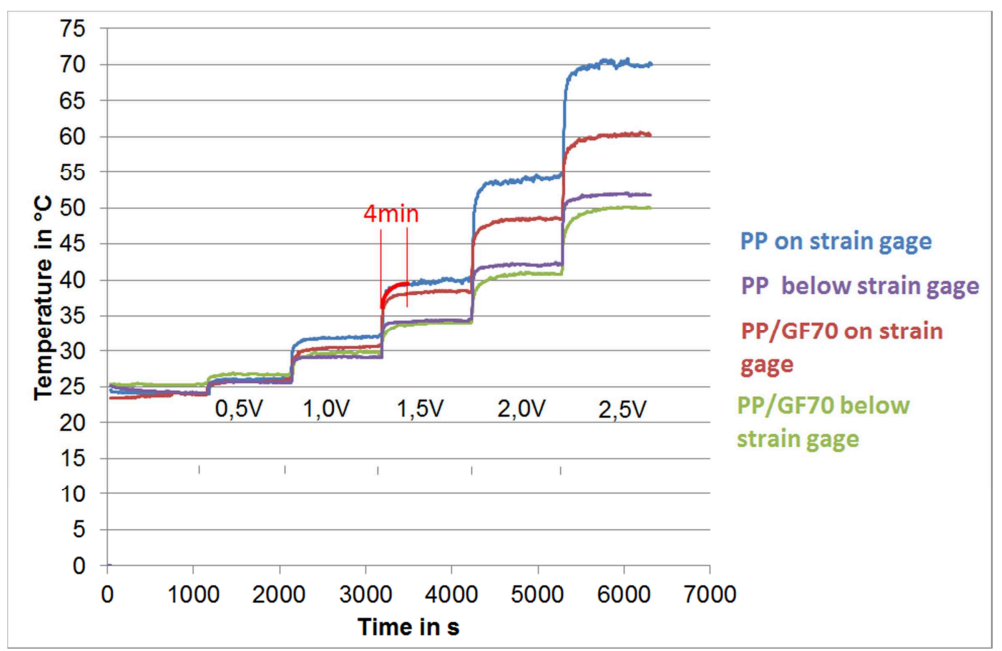

Fig. 3 Temperature rise because of different excitation voltages 
For measuring of the released strains for the residual stress evaluation an excitation voltage of $0.5 \mathrm{~V}$ was chosen. For $1 \mathrm{~V}$ the resulting temperature rise is already about a few degrees, which may already affect the material properties of the polypropylene and therefore influences the strain measurements. The rise of the temperature to a steady state at each stage takes about four minutes. This represents the minimum waiting time before the hole-drilling procedure can be started.

Influence of the material relaxation Thermoplastic materials exhibit a typical relaxation behavior related to the release of the strains here caused by the hole-drilling process. Therefore the relaxation behavior of the four materials has been evaluated by monitoring the time-dependent change of the measured strain signal to a steady state. The results are shown in Figures $4 \mathrm{a}-\mathrm{d}$ for the hole-drilling depth of $0.5 \mathrm{~mm}$, determined using the strain gage with the highest strain signal of every strain gage rosette.

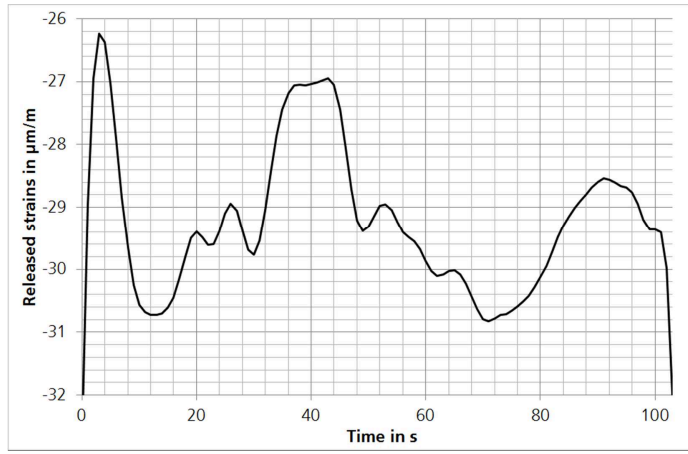

Fig. 4a Strain relaxation measured in PP

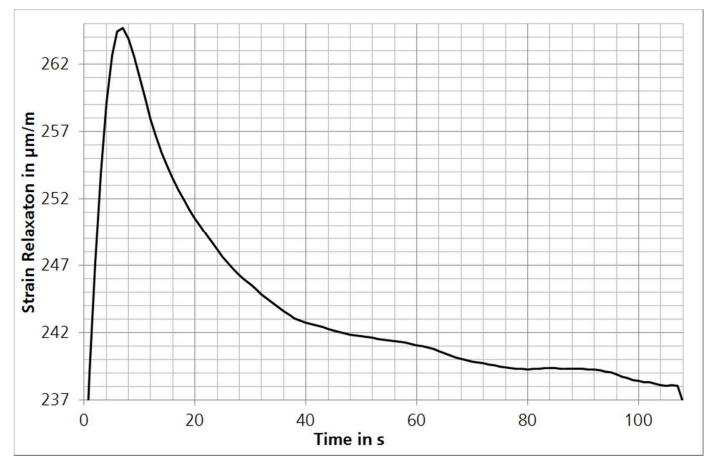

Fig. 4c Strain relaxation measured in PP/GF70 unidirectional laminate - fiber direction perpendicular to the long axis of the component

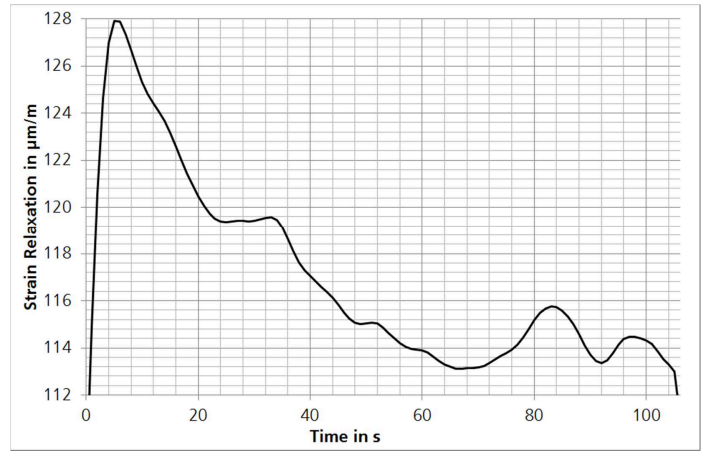

Fig. 4b Strain relaxation measured in PP/GF70 unidirectional laminate - fiber direction parallel to the long axis of the component

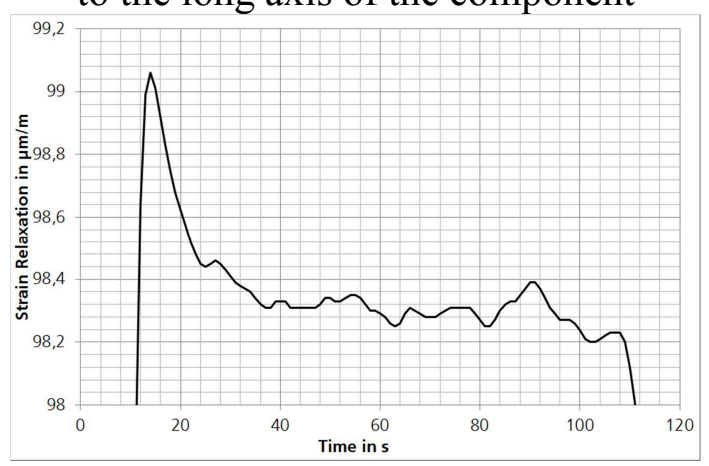

Fig. 4d Strain relaxation measured in the crossply laminate

The polypropylene and the cross-ply laminates exhibit a relatively fast relaxation behavior, as can be seen in the change of released strain below $5 \mu \mathrm{m} / \mathrm{m}$ for the pure propylene and $1 \mu \mathrm{m} / \mathrm{m}$ for the cross-ply laminate in the first seconds. The PP-side of the PP/GF70 unidirectional laminates indicates a longer relaxation time independent of the fiber orientation. The waiting time between the successive drilling steps was therefore adjusted to 100 seconds according to a low slope of the strain relaxation curve of the materials.

\section{Released strains in the components}

In Figures 5 a-d the results of the released strains in the hole-drilling procedure are shown. The diameter of the hole was $1.6 \mathrm{~mm}$. The increment for every depth step was $0.05 \mathrm{~mm}$. The data which is shown is the raw data without the use of fitting procedures.

The pure polypropylene and cross-ply laminates exhibit quite small released strains in comparison with strains measured by means of the hole-drilling in the PP-side of the unidirectional 
laminates. The released strains in fiber direction of the laminates are large in comparison with the other directions.

In the cross-ply laminate the change of the layer direction at a depth of $0.25 \mathrm{~mm}$ and $0.5 \mathrm{~mm}$ can directly be derived from the measured strain signal. On the other hand the maximum measureable depth in the laminate can indirectly be estimated, because the inflection points at the changes of the layer orientations at $0.75 \mathrm{~mm}$ and $1.0 \mathrm{~mm}$ are not visible in the measured strain signals.

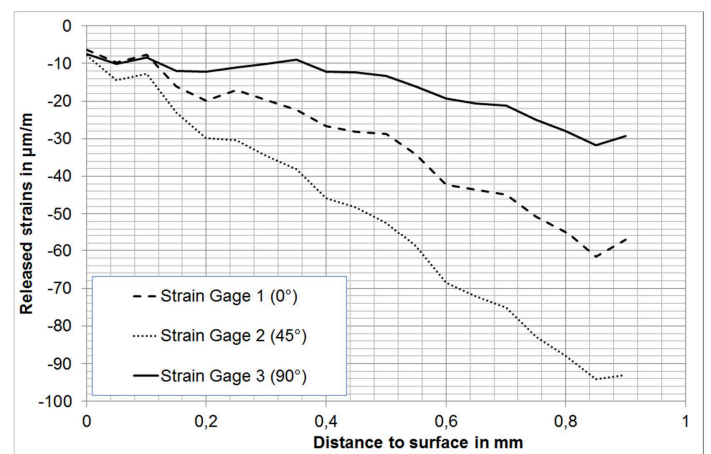

Fig. 5a Released strains in PP

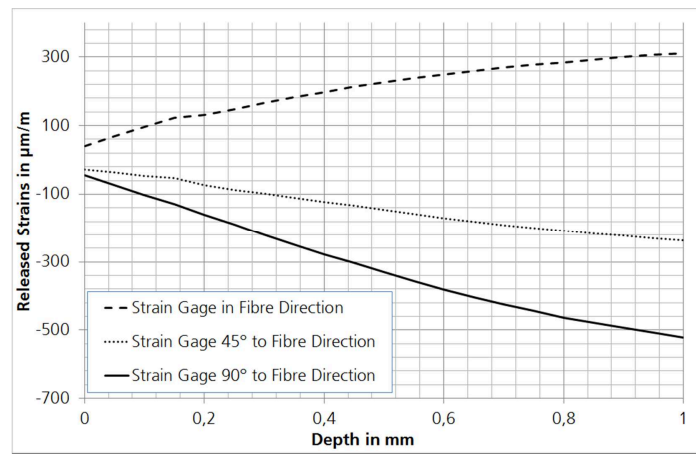

Fig. 5c Released strains PP/GF70 fiber direction perpendicular to the long axis of the component

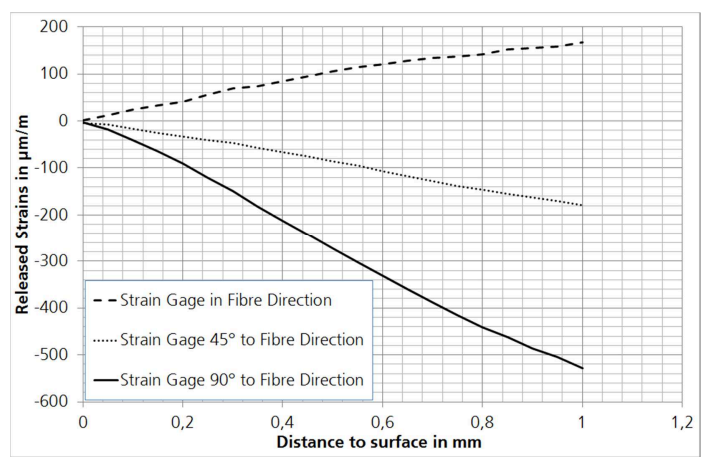

Fig. 5b Released strains PP/GF70 fiber direction parallel to the long axis of the component

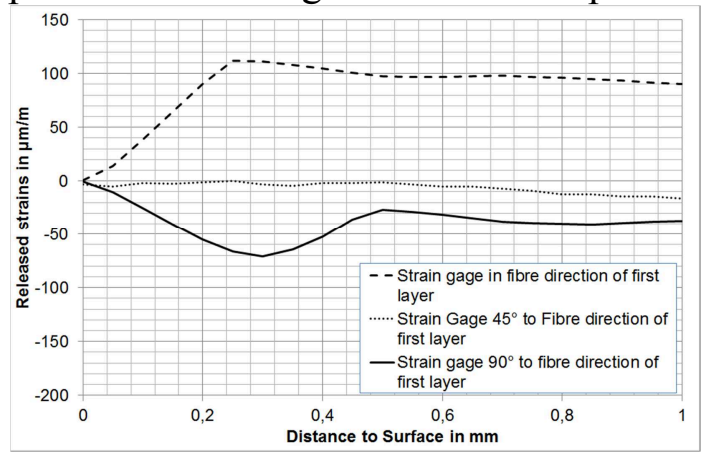

Fig. 5d Released strains in the cross-ply laminate

\section{Calculation of the Coefficient Matrix}

The released strain in the direction of each single strain gage is measured in the respective radial direction $\varepsilon_{\mathrm{r}}$ of the drilled hole [1][2]

$$
\varepsilon_{r}=A\left(\sigma_{x}+\sigma_{y}\right)+B\left(\sigma_{x}-\sigma_{y}\right) \cos 2 \theta+C \tau_{x y} \sin 2 \theta
$$

In matrix form for the strain gage rosette in Fig. 2a equation (1) can be expressed [1] as

$$
\left[\begin{array}{ccc}
A+B & 0 & A-B \\
A & C & A \\
A-B & 0 & A+B
\end{array}\right]\left[\begin{array}{c}
\sigma_{x} \\
\tau_{x y} \\
\sigma_{y}
\end{array}\right]=\left[\begin{array}{c}
\varepsilon_{1} \\
\varepsilon_{2} \\
\varepsilon_{3}
\end{array}\right]
$$

$\sigma_{\mathrm{x}}, \sigma_{\mathrm{y}}$ and $\tau_{\mathrm{xy}}$ are the stresses in and perpendicular to the fiber direction and the shear stress. $\theta$ is the angle of the stresses with respect to the first strain gage.

To calculate the coefficient matrix the four specimens (PP, two unidirectional and one crossply laminate) were modelled using three-dimensional continuum elements in the finite element code Abaqus 6.13. To model the removal of material due to the hole-drilling process the respective finite elements were removed by decreasing the magnitude of their stiffness to $10^{-6}$ of the initial elasticity value. The numerically released strains were calculated as averaged nodal values and integrated over an area equally to the area of the strain gages positioned on the real components. 
In the calculation the polypropylene was modelled using isotropic material properties, whereas for the glass-fiber reinforced composites orthotropic material properties were used. These properties were estimated by the use of the Halpin-Tsai equations [3]. In Table 1 a summary of the material properties is given.

Table 1: Material properties used in the Finite Element Analysis

\begin{tabular}{|l|l|l|l|}
\hline & PP & GF70 in Fiber Direction & $\begin{array}{l}\text { GF70 Perpendicular to the Fiber } \\
\text { Direction }\end{array}$ \\
\hline Type & Isotropic & Orthotropic & Orthotropic \\
\hline Young's Modulus & $1392.3 \mathrm{MPa}$ & $39794.6 \mathrm{MPa}$ & $6068.7 \mathrm{MPa}$ \\
\hline Poisson Ratio & 0.35 & $\begin{array}{l}0.2785 \mathrm{fiber} / \mathrm{matrix} \\
0.45 \mathrm{matrix} / \mathrm{matrix}\end{array}$ & $\begin{array}{l}0.2785 \mathrm{fiber} / \mathrm{matrix} \\
0.45 \mathrm{matrix} / \mathrm{matrix}\end{array}$ \\
\hline Shear Modulus & - & $1779.9 / 2092.7 \mathrm{MPa}$ & $1779.9 / 2092.7 \mathrm{MPa}$ \\
\hline
\end{tabular}

The matrix coefficients in (2) were obtained for every depth step by applying known constant stresses in the direction of the $0^{\circ}$ - and $90^{\circ}$-gages and a shear stress.

\section{Calculation of residual stresses from the measured strains}

In Figures 6 a-d the calculated results of equation (2) for the four components using the data from the strain measurements are shown. The measurement data were fitted by a polynomial function of the $5^{\text {th }}$ grade. The fitting procedure was not applied for the cross-ply laminate, because no appropriate polynomial fitting between $4^{\text {th }}$ and $6^{\text {th }}$ grade could be performed.

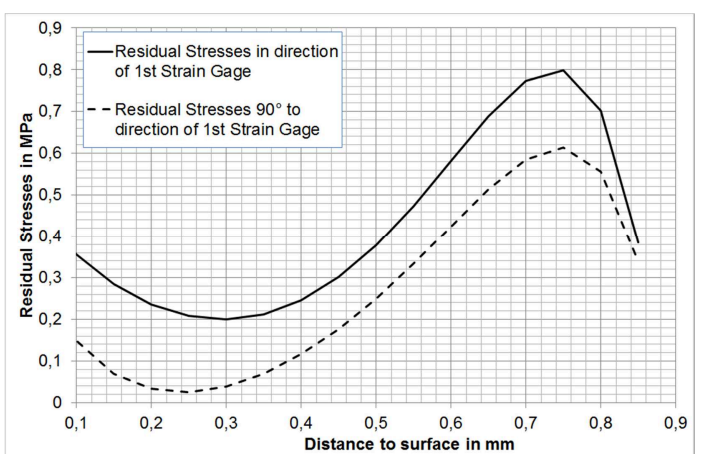

Fig. 6a Calculated residual stresses in PP

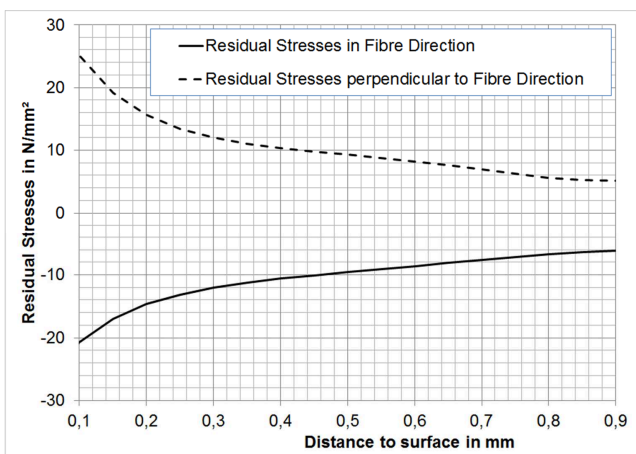

Fig. 6c Calculated residual stresses in the PPside of the unidirectional laminate, fiber direction parallel to the long axis of the component

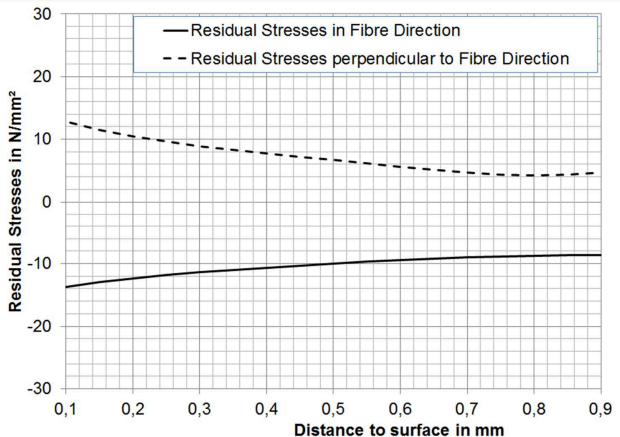

Fig. 6b Calculated residual stresses in the PPside of the unidirectional laminate, fiber direction perpendicular to the long axis of the component

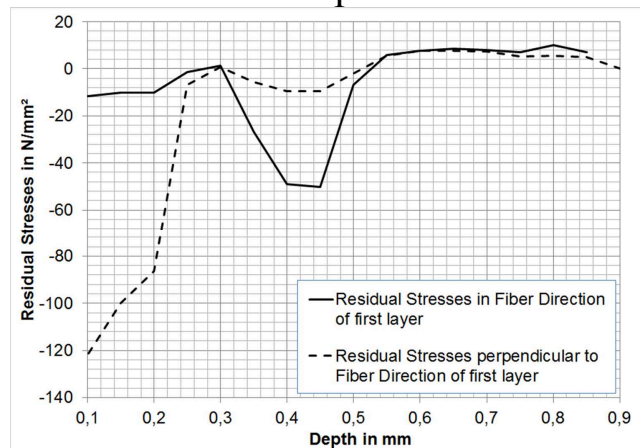

Fig. 6d Calculated residual stresses in PP/GF70 cross-ply laminate 
The pure polypropylene exhibits low tensile stresses. Relatively high compression stresses in the fiber and relatively high tensile stresses perpendicular to the fiber direction can be calculated for the PP/GF70 unidirectional laminates when drilling into the PP-side of the material. The stresses at the surface are higher in the component with the fiber direction parallel to the component, which corresponds to the lower deformation as can be seen in Fig. 1. High values of residual stresses and high gradients can be found in the cross-ply laminate, especially in the direction perpendicular to the fiber direction. It is assumed that because of the high gradients, the missing fitting procedure and the high uncertainties as shown above, these values are considerably over-estimated.

\section{Summary}

The incremental hole-drilling method in thermoplastic materials made of polypropylene and 70 weight-percent glass-fiber reinforced composites can be applied after an adaption of the incremental hole drilling procedure and the analysis method. Following an analysis of different influencing factors the correct boundary conditions for the process could be derived. Large strain measurement uncertainties were observed at drilling speeds below $500 \mathrm{rpm}$. A maximum excitation voltage of the strain gages of $0.5 \mathrm{~V}$ and a minimum waiting time of 4 minutes before the measurement can be started has been derived. The relaxation behavior of the different materials was assessed. The maximum evaluable drilling depth is limited by the strain gage signal. It is quite small in the crossply laminates compared to the evaluable hole drilling depth in the pure polypropylene and the polypropylene part of the PP/GF70-laminates. The evaluation of residual stresses could be performed using an Finite Element Analysis for the determination of the coefficients of the compliance matrix of the isotropic and orthotropic materials. The drilling into the PP-part of the PP/GF70-laminates as well as into the cross-ply laminates leads to the calculation of relatively high residual stresses with respect to the mechanical strength of the materials.

\section{References}

[1] G.S. Schajer, L. Yang, Residual-stress Measurement in Orthotropic Materials Using the HoleDrilling Method, Experimental Mechanics (1994) 324-333.

[2] A.G. Ghasemi, M. Akbarzadeh, Calibration Coefficients for Residual Stress Measurement in Composite Materials Using Finite Element Method, American J. of Engineering and Applied Sciences 5(1) (2012) 25-28

[3] J.C. Halpin et al., The Halpin-Tsai Equations: A Review, Polymer Engineering and Science 16 (1976) 205-207

[4] O. Sicot, X.L. Gong, A. Cherouat, J. Lu, Determination of Residual Stress in Composite Laminates Using the Incremental Hole-Drilling Method., Journal of Composite Materials 37(9) (2003) 831-844

[5] A. Niku-Lari, J. Lu, J.F. Flavenot, Measurement of Residual-Stress Distribution by the Incremental Hole-Drilling Method, Experimental Mechanics (1985) 175-185 